\title{
De la crisis fordista a la crisis de la financiarización: la centralidad del espacio en la crisis actual. Madrid, 1985-2007
}

\author{
Alexandra Delgado Jiménez \\ Universidad Politécnica de Madrid, España. Email: alexandra_delgado@hotmail.com
}

\begin{abstract}
Resumen: Se analiza la incorporación del espacio a las dinámicas centrales de los procesos políticos y económicos en el último cuarto del siglo XX en España, a través de su relación con las sucesivas crisis, principalmente reconocidas por su componente económico. Se ha apostado por la producción de espacio (transformación y crecimiento) como respuesta o salida a la crisis fordista, cuestión que finalmente se ha convertido en un componente principal dentro de la actual crisis de financiarización. Se toma como ejemplo el caso de Madrid por resultar paradigmático, ya que la región ha desempeñado un papel como laboratorio de nuevas formas de gobierno y planificación que han dado un mayor protagonismo al espacio, principalmente por el apoyo al crecimiento físico, como motor de crecimiento económico y de competitividad de la región metropolitana en el concierto europeo y mundial de ciudades.
\end{abstract}

Palabras clave: crisis, realidad socio-espacial, modelo, gobierno.

\section{From fordist crisis to the crisis of financialization: the centrality of space in the current crisis. Madrid, 1985-2007}

\begin{abstract}
The incorporation of the space to the central dynamics of the political and economic processes is analyzed in the last quarter of XXth century in Spain, through its relation with the successive crisis, mainly recognized by its economic component. The production of space (transformation and growth) has become one of the key answers to the fordist crisis. This question has finally become a main component in the current crisis of financialization. By way of example, it is used the case of Madrid, because it is considered paradigmatic, since the region has played a leading role as a laboratory for new forms of governance and planning, which has given a centrality to space, with the increase of physical growth, as motor of economic growth and competitiveness in the metropolitan region of Madrid in the context of European and international cities.
\end{abstract}

Key words: crisis, socio-spatial reality, model, government.

\section{Da crise fordista para a crise da financeirização: a centralidade do espaço na atual crise. Madrid, 1985-2007}

Resumo: Discute-se a incorporação do espaço central para a dinâmica dos processos políticos e económicos no último quarto de século, em Espanha, através 
de sua relação com as crises sucessivas, conhecida principalmente pela sua componente económica. Optou-se pela produção do espaço (de transformação e crescimento) em resposta ou da crise fordista, uma questão que, em última análise se tornou um componente importante na crise da financeirização. Toma-se o exemplo de Madrid como paradigmático, já que a região tem desempenhado um papel como um laboratório para novas formas de governança e de planejamento que tem dado um papel mais importante no espaço, principalmente através do apoio ao crescimento físico, como motor de crescimento e da competitividade económica da região metropolitana no concerto europeu e mundial da cidades.

Palavras-chave: crise, modelo sócio-espacial, governo.

$$
* * *
$$

"Es importante comprender que el espacio no es neutro ni inocente sino que es por excelencia uno de los campos de acción de las fuerzas políticas:

el estado es también una entidad geográfica y el aparato de estado organiza el espacio geográfico de modo de ejercer su poder sobre los hombres"

Yves Lacoste, La Philosophie des sciences sociales. Hachette, 1973. París. Citado en Lipietz, 1979:173

\section{Introducción}

En las últimas décadas del siglo XX se han vivido diversos ajustes del modelo económico. Éste se ha reconfigurado a través de las respuestas políticas adoptadas como salida a las sucesivas crisis de acumulación, propias, por otra parte, del sistema económico imperante, el sistema capitalista.

Una de las principales transformaciones que se han vivido en dicho periodo ha sido la creciente urbanización a nivel mundial. El mundo se ha hecho más urbano en cuanto población -en 2007, la mitad de la población mundial ya era urbana, según datos de UN-HABITAT-, pero también y, sobre todo, se ha desarrollado la dimensión espacial del modelo económico, desdibujando las características que podrían definir a lo urbano (cierta densidad y configuración que favorecen la accesibilidad, información e innovación, entre otras cuestiones), lo que ha fomentado en última instancia el desarrollo de la suburbanización.

Cabe destacar la centralidad adquirida por el espacio: el futuro de la Humanidad se encuentra en "su" espacio, es decir, en el medio ambiente (Lipietz, 1990:2). El espacio a tomar como el medio ambiente ha cobrado protagonismo máximo -hasta el punto que se considera el gran problema 
actual- e indica la necesidad de buscar alternativas al modelo ya que parafraseando al científico Albert Einstein «los problemas no pueden resolverse con las mismas lógicas que los crearon».

Ante estas cuestiones, se centra el análisis en cómo el espacio se ha incorporado a las lógicas centrales del modelo adoptado para la salida de la crisis en los países desarrollados por la importancia adquirida por el circuito secundario de acumulación, es decir, el espacio construido.

\section{Capitalismo y espacio en el contexto histórico}

El sistema capitalista necesita la explotación de recursos para incorporar al ciclo de acumulación de capital, así como un entorno no capitalista para la reproducción social.

La teoría marxista es de carácter teórico y, por tanto, no introduce ni el espacio ni el tiempo histórico, ya que trata de ser general. No inscribe los procesos que explica en un espacio y tiempos concretos, pero sí introduce el tiempo teórico de explicación de los procesos capitalistas.

El espacio se incorporó posteriormente en la medida en que se habló de imperialismo, como en el libro de Lenin El imperialismo, fase superior del capitalismo (Lenin, 1916), entre otros.

Lipietz indica en su obra El capital y su espacio: "Marx y Engels no hablan del espacio más que a propósito de los orígenes del modo de explotación capitalista: la división del trabajo, la separación ciudad-campo” (Lipietz, 1979: 11). Y también aclara a continuación que "como no es ése el objeto de la teoría marxista, no es nada sorprendente que hasta ahora no se haya ocupado, según parece, de esos problemas (del mismo modo que, por ejemplo, no se ha ocupado hasta fecha reciente del problema de la moneda)".

El espacio ha sido tomado generalmente como el telón de fondo de los acontecimientos, donde la única dimensión que había sido considerada para la Historia era el Tiempo (Lipietz, 1990:1). El autor declaraba anteriormente en este sentido: "Toda realidad material existente (y toda relación social tiene una forma de existencia material) tiene una dimensión espacial y una dimensión temporal” (Lipietz, 1979: 19).

En los años setenta se empezó a hablar de capitalismo y espacio, entendido este último como espacio físico, no sólo factor de localización sino también superficie consumida, lo que se relaciona con la situación de continuo crecimiento, desde la Segunda Guerra Mundial, y con la emergente preocupación medioambiental. El informe del Club de Roma (Meadows et al, 1972) sobre los límites al crecimiento, es un referente de la relación que denunciaba en la época el modelo de desarrollo basado en el crecimiento y los impactos en el medio ambiente. 
El filósofo francés Henri Lefebvre, que relacionó el espacio y su producción, en lo que llamó la reproducción de las relaciones sociales de producción, apuntó que el capitalismo se reproduce consumiendo espacio (Lefebvre, 1976), o más concretamente que "sobrevive a través de la producción de espacio, pero no explicó exactamente cómo sucedía esto” (Harvey, 2004: 99), lo que ha supuesto un enigma a desentrañar.

Esto plantea, a priori, que las condiciones de la reproducción ampliada del capital provocan fuertes contradicciones, y que al revolucionar permanentemente el modo de producir y el modo de consumir, revolucionan consecuentemente la organización del espacio (Lipietz, 1979: 8).

Tras estas ideas, se han formulado desarrollos teóricos que tratan de explicar el papel del espacio en el sistema capitalista, destacando las aportaciones de Harvey.

Harvey, influenciado por Lefebvre como otros investigadores de de la teoría urbana actual, principalmente dentro de la geografía humana, retoma la afirmación de Lefebvre de que el capitalismo se reproduce consumiendo espacio e introduce un nuevo término para determinar la relación entre el sistema capitalista y el espacio: el ajuste espacio-temporal (Harvey, 1982; 2004; 2007). Este arreglo consiste en dos mecanismos espaciales para salvar las crisis de sobreacumulación: la expansión geográfica y la reorganización espacial, es decir, la producción de espacio ya sea por crecimiento o por transformación. Se trata de utilizar intensivamente el circuito secundario de acumulación capitalista, el espacio construido, para resolver las crisis capitalistas.

Existen muchos mecanismos por los cuales a través del espacio se pueden obtener plusvalías -de la renta del suelo-, y en todo caso, retrasar lo que incorpora el factor tiempo- las crisis de sobreacumulación. En el caso del crecimiento, ha destacado principalmente la escala de los procesos o el cambio de escala y volumen que se ha alcanzado, de carácter monetario de los préstamos hipotecarios, cemento consumido, etc., destacando el valor que se le ha dado al espacio construido como valor de cambio (ver figura 2). Se conforma, por ello, un espacio de acumulación de capital, de activos financieros donde no importa la calidad espacial-ambiental.

Otro de los mecanismos claves de los ajustes espacio-temporales son los que buscan, como su propio nombre indica, el aplazamiento temporal de las contradicciones que derivan del exceso de producción -y la consecuente caída de la tasa de beneficios-, unido en ocasiones a la expansión geográfica, ya que ésta implica inversiones a largo plazo en infraestructuras cuyo valor tarda en desarrollarse.

En los años noventa, desde la escuela regulacionista, Lipietz apuntó una de las características que tiene el espacio respecto el capitalismo, o más concretamente, el entorno no capitalista: la “estructuración del espa- 
cio” es una de la dimensiones materiales de la estabilización de las relaciones estructurantes de las prácticas sociales. Esta estructuración del espacio es a primera vista el resultado de esta estabilización y se convierte a posteriori -o a priori, en procesos planificados- en la base material de la estructuración social. La infinita plasticidad de las prácticas sociales queda enmarcada, "rigidizada”, “cosificada”, como diría Karl Marx, lo que se convierte en un modo de regulación del espacio humano (Lipietz, 1990:4).

Esta estructuración del espacio tiene un papel importante en la reproducción social ya que limita en cierto grado las transformaciones sociales. Resulta, por tanto, que el futuro del espacio (urbano) debe tener en cuenta las consideraciones sobre la evolución social global y el modo en el que se reestructura el espacio, y también la manera en la que el espacio heredado limita y condiciona la evolución social. Según las propias palabras de Marx "los hombres hacen su propia historia, pero sobre la base de las condiciones heredadas del pasado” (Lipietz, 1979:8).

El futuro del espacio no es la simple proyección sobre el terreno del futuro de la sociedad. La sociedad existe como una forma del territorio y por tanto, el espacio humano es una coacción para la sociedad futura y una base de partida.

La contradicción entre los espacios existentes, que por una parte materializan las formas de la civilización que han existido hasta ahora y los “espacios proyectados” con la materialización de modelos de desarrollo en competencia para el futuro, debería estar regulada (Lipietz, 1990:5).

Estos mecanismos - el ajuste espacio-temporal para salvar las crisis capitalistas y la estructuración del espacio como estabilización social - demuestran la clara relación que existe entre el desarrollo capitalista y la producción de espacio, en sus versiones de transformación y crecimiento.

\section{Procesos espaciales contemporáneos}

Además de la escala de los procesos de urbanización, destaca el surgimiento e intensificación de algunos procesos de transformación urbana en el interior de las ciudades. Existen numerosos ejemplos de transformación urbana de las ciudades existentes en todas partes del mundo, desde la ciudad de Cartagena de Indias en Colombia a Cracovia en Polonia. Madrid también es ejemplo de eso con la remodelación por su mayor revalorización del eje Prado-Recoleto donde se encuentra el Triángulo del Arte, con los museos del Prado, Reina Sofía y Thyssen-Bornemisza.

Entre los procesos espaciales contemporáneo destacan los mecanismos de gentrificación o elitización, en cuanto a la generación de la plusvalía por la reorganización social y espacial y la museificación y tematización, 
en cuanto a la intervención/reorganización espacial para convertir a las ciudades en objeto del turismo de masas, es decir, en espacios de consumo (Montaner, 2003).

La gentrificación o elitización consiste en un proceso de transformación urbana en el que la población de un barrio deteriorado es progresivamente desplazada por otra de un mayor nivel adquisitivo a la vez que éste se renueva (aquí se podría hablar de si es el motivo o la conclusión). La tematización, por su parte, es “dedicar partes acotadas, comprensibles y completas de ellas mismas a un tema concreto, que se ofrece como atractivo al turismo local e internacional [...] lo que implica simplificar la complejidad de la propia historia para ofrecer un discurso simple y transmisible” (Montaner, 2003). Dentro de este tipo cabe destacar el análisis realizado sobre la urbanalización, término introducido por Muñoz (2004), que habla de la simplificación progresiva del hecho urbano y de su nueva naturaleza genérica, multiplicada y a la vez única, que caracteriza el espacio urbano actual Por último, definir la museificación como el embalsamamiento en su estado histórico de una ciudad o una parte de ella, limitando sus propias dinámicas con la finalidad de hacer atractivo ese espacio como objeto de consumo.

\section{De la crisis fordista a la crisis de la financiarización}

Algunos expertos hablan de cómo las crisis económicas que se han vivido en este periodo son ciclos propios del sistema capitalista, fases B de las ondas de Kondratieff. Esta observación apareció por primera vez en el libro de Kondratiev (1925) The major Economic Cycles, y fue seguido de un libro de Joseph Schumpeter (1939), que sugirió llamarlas así, ondas de Kondratiev.

Pero también hay expertos que apuntan que por la profundidad y duración de las últimas crisis éstas podrían suponer el final del sistema capitalista, o incluso formar parte de un periodo de transición - como ondas de transición del nuevo ciclo, lo que explicaría la creciente cercanía de las mismas - en el que podríamos estar inmersos. Esta cuestión no podría dilucidarse hasta pasado cierto tiempo, como apunta el sociólogo experto en economía, Isidro López ${ }^{1}$. Estas ideas se desarrollan en los textos de Arrighi (2008), Adan Smith en Pekín, y de Wallerstein (2007), Geopolítica y geocultura: ensayos sobre el moderno sistema mundial.

Una de las características del periodo principal de análisis 19852007, es que está enmarcado por la existencia de dos crisis. En primer lugar, la crisis fordista (unida al modelo de producción keynesiano) y desde el año 2007 y en la actualidad a la crisis de la financiarización.

El modelo fordista era un modelo de regulación de un modo de producción, el keynesianismo, basado en la demanda, y un entorno no capita- 
lista en el que se apostaba por la planificación de las relaciones estructurales, a través de la legislación social, las convenciones colectivas y el estado, en su papel de Estado Benefactor.

Este modelo de producción keynesiano-fordista, es la base de la jerarquización funcional que ha habido en el seno de las ciudades entre los barrios (o distritos) y en otra escala entre el campo y la ciudad. Ésta es una de la huella más importante que ha dejado la denominada "modernidad”.

El modelo fordista entró en crisis por diversos factores como la crisis de acumulación, la desestabilización que supuso el concurso internacional en este modelo, que había funcionado dentro de los Estados Unidos y la excesiva rigidez que suponía, entre otros.

Le sucedió el denominado postfordismo que se caracterizaba por los cambios en la división internacional del trabajo y la globalización de las finanzas, tras la cual se inició un proceso que algunos expertos han denominado financiarización de la economía: “en este marco, el síntoma principal de la economía actual, la financiarización, o si se quiere el específico resumen de la acumulación de capital sin mediación de la producción de mercancías, reflejaría simplemente este creciente desafía la realización de valor en términos capitalistas. Bajo esta perspectiva, la financiarización es sólo la prolongación de una solución, en principio temporal y transitoria, a los problemas de la economía en general [...] se trata de los problemas de realización del valor en términos capitalistas y de la recuperación de unas tasas de beneficios que en las últimas décadas han experimentado trayectorias erráticas y mucho menos vibrantes que las de épocas pasadas” (López y Rodríguez, 2010:30).

En otras palabras, el nuevo modelo de financiarización se basa no en la capacidad productiva del modelo capitalista sino en la capacidad de financiación, cuestión que hasta ese momento había sido auxiliar.

La globalización de los mercados financieros y la financiarización de la economía han sido cuestiones centrales del nuevo modelo y también centrales en la crisis.

Este modelo de financiarización se apoyó en la incorporación del espacio a través de la construcción de obra nueva y especialmente la vivienda que se consideró un activo.

Se observa cómo la producción de viviendas se incrementó hasta en más de dos veces desde los años 90, época en el que se empezó a fomentar la financiarización de la economía. En cuanto a vivienda libre, la proporción es mucho mayor llegando a ser más de cuatro veces, respecto a la proporción de viviendas terminadas de los años ochenta (ver figura 1, página siguiente). 
Figura 1. Viviendas terminadas en España: protegidas y libres, 1964-2008.

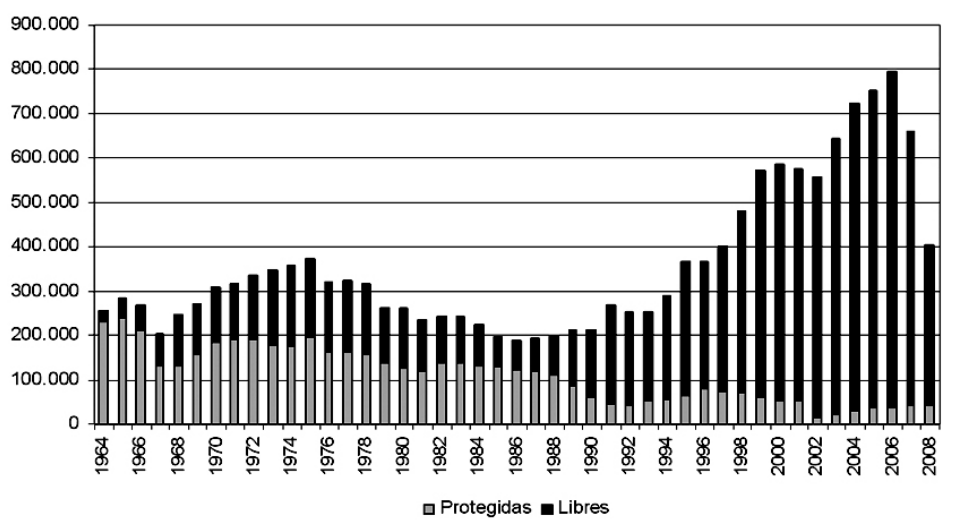

Fuente: Elaboración propia a partir de Bases de Datos Histórica del BBVA y Ministerio de Vivienda.

Nota: Los datos de viviendas a partir de 1992 son de viviendas iniciadas, según las Estadísticas del Ministerio de Vivienda.

Para ver la escala de los procesos en el sector inmobiliario y su relación con la financiarización, se analizan conjuntamente las viviendas iniciadas, el consumo aparente de cemento -se puede considerar que es un buen indicador de artificialización- y el volumen monetario de los préstamos hipotecarios a vivienda en el periodo 1995-2008 (figura 2).

Figura 2. Evolución de las viviendas iniciadas, el consumo de cemento aparente y el volumen monetario de los préstamos hipotecarios a vivienda en España, 1995-2008. (1995=base 100).

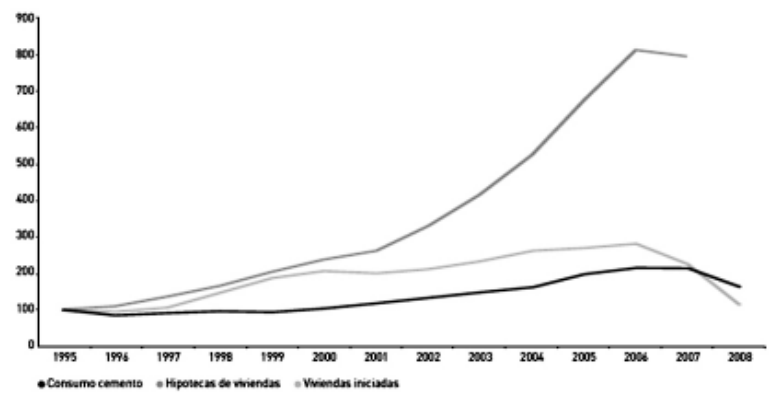

Fuente: OSE (2009) a partir de datos de Colegio de Arquitectos de España, INE y OFICEMEN. 
En España, se detecta un aumento desmedido de las viviendas iniciadas en el periodo de estudio, sobre todo entre los años 2001 y 2007. En 2008 descendió ligeramente por primera vez en el periodo. El aumento acumulado desde 1995 a 2007 es de un 226\%, en estrecha relación con el consumo aparente de cemento -de $214 \%$ en 2007 y cuyo máximo se alcanzó en 2006-.

En 2008, se produjo un fuerte descenso de ambas magnitudes, llegando a 114\% respecto a niveles de 1995 en el número de viviendas iniciadas y a $163 \%$ en el consumo aparente de cemento.

Pero el aumento más significativo es el del volumen monetario de los préstamos hipotecarios, que en 2007 era ocho veces superior a 1995 (en concreto 793\%). Se puede considerar por tanto que ha habido una fuerte financiarización del sector inmobiliario, siendo la proporción de las hipotecas respecto a las viviendas iniciadas de cuatro a uno (OSE, 2009).

En el caso de España, este modelo ha entrado en crisis recientemente a partir de 2007.

\section{El contexto de crisis en España: 1973-1984, 1993-1995 y desde 2007}

Resulta de gran interés mostrar las características y salidas adoptadas de las sucesivas crisis que se han vivido en España desde el comienzo de la democracia - la mayoría de carácter internacional-, ya que éstas han marcado decididamente la agenda política y han mostrado las inconsistencias del modelo de desarrollo adoptado.

Desde un punto de vista convencional, y tomando como indicador el PIB -porque todas las crisis a analizar tienen en común la componente económica- se puede apuntar que las crisis que se han sucedido en España se corresponden a los periodos 1973-1984, 1993-1995 y desde el año 2007. Cabe destacar que los periodos 1973-1984 y el último, desde 2007, no comienzan con PIB negativo como el periodo 1993-1995, pero muestran un fuerte descenso, llegando rápidamente a PIB negativo. En el caso del primer periodo analizado, 1973-1984, muestra fuertes altibajos en el PIB con tendencia decreciente (Ariño, 1997).

Cabe destacar que estas crisis se dan en tres contextos muy diferenciados si los analizamos respecto a la relación económica de España con el contexto territorial más cercano, esto es, la Unión Europea, y en un marco mayor, en los países occidentales y desarrollados.

En la primera crisis analizada, 1973-1984 España no había entrado a formar parte de la Unión Europea y tenía pleno dominio sobre las políticas, en especial, la economía.Esta crisis es considerada junto a la Gran 
Figura 3. Tasa de crecimiento del PIB en España, 1971-1996.

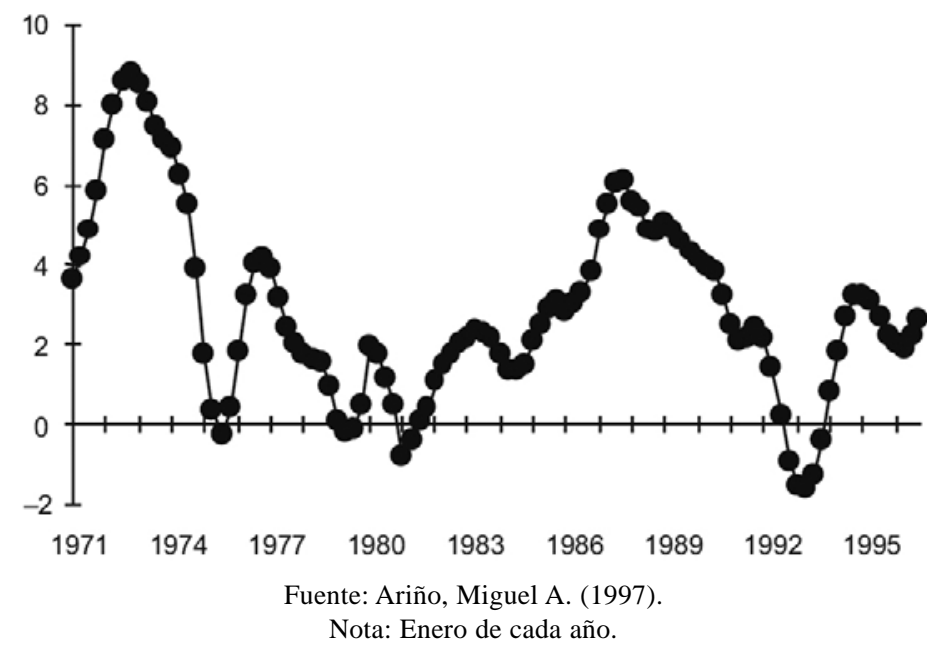

Figura 4. Tasa de crecimiento del PIB en España, 1997-2009*.

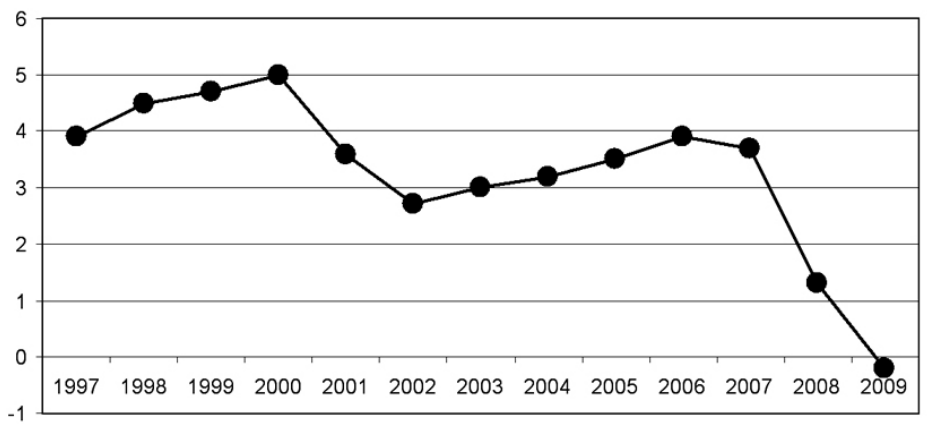

Fuente: Elaboración propia a partir de datos de Eurostat. Nota: Dato 2009, previsión.

Depresión - tras el crack del 29- y la crisis actual, una de las más importantes de la época reciente.

La crisis de 1973 tuvo su antecedente en el desorden del sistema monetario internacional, precipitado por la caída del dólar. La crisis del petróleo de 1973 comenzó a partir del 17 de octubre de 1973, a raíz de la decisión de la Organización de Países Árabes Exportadores de Petróleo (que agrupaba a los países miembros árabes de la OPEP más Egipto y Siria), 
anunciando que no exportarían más petróleo a los países que habían apoyado a Israel durante la guerra del Yom Kippur, que enfrentaba a Israel con Siria y Egipto. Esta medida incluía a Estados Unidos y a sus aliados de Europa Occidental.

Al mismo tiempo, los miembros de la OPEP acordaron utilizar su influencia sobre el mecanismo que fijaba el precio mundial del petróleo para cuadruplicar su precio, después de que fracasaran las tentativas previas de negociar con las «Siete Hermanas».

El aumento del precio unido a la gran dependencia que tenía el mundo industrializado del petróleo de la OPEP, provocó un fuerte efecto inflacionista y una reducción de la actividad económica de los países afectados. Estos países respondieron con una serie de medidas permanentes para frenar su dependencia exterior.

En la segunda crisis, 1993-1995, España ya formaba parte de la Unión Europea, tras la firma del Acta de Adhesión de España a las Comunidades Europeas el 12 de junio de 1985. Esto le obligaba a seguir las Directivas Europeas, pero todavía conservaba la potestad sobre la moneda, capacidad que usó al devaluarla - en concreto en tres ocasiones, el 16 de septiembre de 1992, en un 5\% y el 21 de noviembre siguiente en un 6\%, y el 13 de mayo de 1993, en un 8\% (De la Osa, 1992)-, para poder conseguir estabilidad económica - ya que de este modo se controla la inflación, se aumentan las exportaciones y se equilibra la balanza de pagos.

Esta crisis se puede considerar de carácter coyuntural, pero de gran importancia respecto a la incorporación del espacio a las dinámicas centrales, ya que sirvió de revulsivo para una serie de toma de decisiones que han configurado un nuevo modelo de desarrollo económico, y por ende, espacial.

Esta crisis, debido principalmente al corto periodo de tiempo, no tuvo una gran incidencia directa en el territorio, principalmente respecto al desarrollo físico, pero sí que fue el contexto en el que se fraguó el cambio de paradigma respecto al territorio y su papel en la economía, y supuso una serie de decisiones sobre planificación espacial, que posteriormente influirían enormemente en el territorio, principalmente en los usos del suelo.

Por último, la crisis que se ha iniciado en 2007, surge en un contexto totalmente diferente, ya que España, además de formar parte de la Unión Europea, se ha sumado a la zona Euro, bajo la dirección del Banco Central Europeo (BCE), lo que principalmente se traduce en la obligatoriedad de asumir el tipo de interés del BCE y en que ya no es posible devaluar la moneda de manera diferenciada al resto de países de la Unión Europea.

Se puede considerar el inicio de la crisis en septiembre de 2007, momento en el que aparecieron los primeras turbulencias en el sistema financiero tras la explosión de las hipotecas subprime en agosto del mismo año. Algunos autores la remontan a abril de 2007, debido a la caída de la 
inmobiliaria española Astroc (ver noticia "La inmobiliaria Astroc pierde un 43\% de su valor en Bolsa en un día”, Cinco Días, 19 de abril de 2007). Otros consideran que la fecha de comienzo a nivel internacional puede ser el 15 de septiembre de 2008 con la caída de Lehman Brothers, el cuarto banco de inversión de Estados Unidos.

Pero aunque se fije el inicio de la crisis por factores financieros, se puede considerar que se trata de una crisis de gran complejidad si la comparamos con las anteriores. Principalmente destaca y así se muestra en todo tipo de medios de comunicación, que ésta es una crisis financiera (Berzona, Rogoff: 2009) y se habla de crisis de confianza (Campuzano, 2008) de las instituciones bancarias, así como de falta de regulación en diferentes momentos de la vida de un crédito - en la concesión, a personas con alto riesgo de impago en lo que se ha denominado hipoteca subprime; en la división de estos créditos y su puesta en circulación en los mercados internacionales, etc.. También se habla, sobre todo en el caso español, como en años atrás ocurrió en Japón, de la crisis del sector de la construcción y de cómo la tan traída y llevada “burbuja inmobiliaria” ha “pinchado”. En palabras de Julio Rodríguez (2008), experto en mercado de vivienda, se trata de muchos más factores: "se superponen varias crisis en la coyuntura presente. La de los mercados de vivienda, la crediticia ya citada, la situación quebrantada de numerosos bancos situados en diferentes países, los crecientes precios de los alimentos y de las primeras materias. Se está produciendo un ajuste macroeconómico global.”

Cabe traducir, en cuanto a la dimensión espacial, que la crisis de los mercados de vivienda es una crisis de sobredimensionamiento de construcción de obra nueva, basado en unas expectativas que se han demostrado irreales y que no se refieren ni relacionan con los límites medioambientales.

\section{La incorporación del espacio a las dinámicas centrales en relación a las crisis: el caso de Madrid}

En España, pero especialmente en Madrid, la crisis económica de los años setenta tuvo una gran incidencia en el territorio, ya que hubo una fuerte remodelación del sistema productivo. Madrid se había convertido en la primera ciudad industrial del país -cuestión de destacar, ya que Madrid no tenía un pasado industrial como en el caso de otras ciudades españolas, como Barcelona o Bilbao-, lo que tuvo su reflejo en el espacio social.

Esta crisis supuso la adopción de muchas medidas en términos espaciales y de planificación. En plena composición de los ayuntamientos democráticos, se apostó por la conservación del suelo productivo -especialmente industrial para evitar pérdida de empleo, aunque ya se había iniciado un proceso de fuerte terciarización-, la recualificación urbana y la participación pública.

En un cambio de política espacial, en relación con la crisis, las políticas financieras se basaron desde entonces en subsidios indirectos, la pro- 
moción del mercado hipotecario, favoreciendo el endeudamiento familiar, e incluso fomentando la segunda residencia.

Se fomentó la promoción del mercado hipotecario a través de ligar a la propiedad a grandes franjas de la población, obteniendo transferencia de rentas hacia finanzas que necesitan liquidez y transferencia de los riesgos de los mercados financieros a las familias; esta política es herencia de los Pactos de la Moncloa, que se firmaron en 1977.

Además, se incorporó al espacio a las dinámicas centrales del modelo a través de subsidios indirectos a la demanda inmobiliaria. El Decreto Boyer de 1985 tiene como objetivo "estimular el consumo privado y la inversión fomentar el empleo e impulsar el sector de la construcción”.

La liquidación del parque inmobiliario en alquiler, también favorecido por el decreto Boyer, está unido a la dependencia de las políticas institucionales al crecimiento económico, y el sostén de la vivienda protegida de los ciclos recesivos del sector de la construcción (ver figura 1), y que se ha sostenido con grandes desarrollos urbanísticos, como en el caso de los Programas de Actuación Urbanística, con los cuales se ha desarrollado grandes bolsas de suelo.

Se trata de un modelo urbano que se apoya en el aumento de la oferta de suelo, y, en última instancia, la oferta de vivienda, con un papel atribuido de activo económico y valor patrimonial de las familias. En la exposición de motivos de la Ley Hipotecaria española vigente se dice que "la vivienda supone la mayor parte del patrimonio de las familias y por tanto debe ser protegido".

\section{Figura 5. Superficie a construir con licencia residencial por comuni-} dad autónoma en España, 2000-2008.

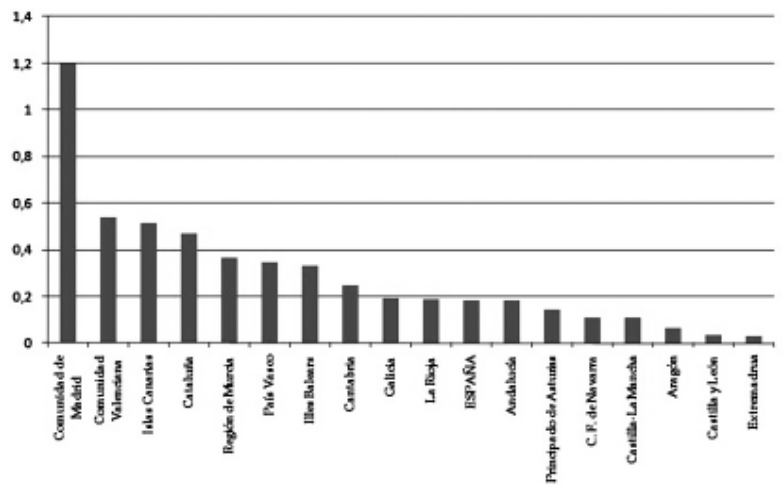

Fuente: Elaboración propia a partir de Ministerio de Fomento (2009). 
Si analizamos los datos del periodo 2000-2008, la superficie ocupada por las viviendas a construir (según licencias) en España fue de un 0,18\% del territorio nacional. La Comunidad de Madrid fue la autonomía que más creció en superficie residencial, con una proporción del 1,20\% respecto a la superficie de la comunidad autónoma, lo representa es más de seis veces la media nacional. Le siguen la Comunidad Valenciana, con un 0,54\% y Canarias, con un 0,52\%. En el extremo opuesto, se encontraban Extremadura $(0,03 \%)$, Castilla y León $(0,04 \%)$ y Aragón $(0,06 \%)$, todas ellas de gran extensión (figura 5) (OSE, 2009).

En 2007 comienza la actual crisis, que se puede denominar crisis de financiarización, en la cual el papel que juega el territorio es clave. En la actualidad, se vuelve a estar en un momento decisivo para reflexionar sobre el papel que ha tenido el espacio y el territorio en el modelo de desarrollo adoptado y, por tanto, para evitar algunos posicionamientos que han propiciado esta situación.

\section{A modo de conclusión}

El espacio ha desempeñado un papel central en el modelo de desarrollo del último cuarto de siglo en España. La apuesta por apoyarse en el consumo espacial, tomando el espacio como consumo de superficie (suelo), ha tenido como consecuencia un mayor impacto ambiental y social de la actual crisis respecto a anteriores.

Debido a la multitud de interacciones que existen entre crisis, realidad socio-espacial, modelo y gobierno, por la adopción de medidas y modelos, se muestran las principales las principales conclusiones en relación a dichos elementos:

- El modelo urbano se ha transformado enormemente en el periodo de estudio, 1985-2007, convirtiéndose en un modelo despilfarrador de recursos, entre ellos el suelo, pues no se basa en las necesidades.

- El gobierno ha optado por poner el espacio (el territorio, el suelo) al servicio de la política económica basada en el crecimiento.

- El territorio ha recibido los numerosos impactos por tomar principalmente la ciudad como motor de crecimiento.

- El gobierno de la ciudad ha sido de carácter intervencionista, tanto en periodos austeros como de crecimiento. En los periodos de crecimiento, la intervención se ha basado principalmente en el apoyo del sector privado para evitar impedimentos que ralentizaran el crecimiento.

- Toda crisis supone, en general, un replanteamiento del modelo. Los periodos de crisis han sido momentos de transformación de las 
políticas (es decir, del gobierno). La respuesta a la crisis de los años 90 ha sido el aumento de la oferta de suelo como fomento de la ciudad como motor de desarrollo y apuesta por una "masa crítica" para acceder al concierto europeo y mundial de ciudades, es decir, al ranking de "ciudades globales".

- Ese posicionamiento a favor del crecimiento ilimitado ha entrado en crisis. La primera cuestión que se ha desmoronado ha sido la capacidad de financiación, a la que han seguido las siguientes, tales como, los instrumentos, el planeamiento y la construcción social de una la expectativa de crecimiento ilimitado, la cual ha entrado en crisis una vez superado el límite de capacidad financiero en lugar de otros límites como los ambientales, muy anteriormente superados.

Y por tanto, se puede concluir que el territorio ha sido puesto al servicio del modelo de desarrollo económico de los últimos años, lo que no ha hecho más que apoyar su incorporación a una escala y velocidad no conocidas, a las dinámicas capitalistas, como circuito secundario de acumulación. 
Polis, Revista de la Universidad Bolivariana, Volumen 10, $N^{\circ}$ 28, 2011

\section{Nota}

${ }^{1}$ Entrevista 8 de septiembre de 2009. 


\section{Bibliografía}

Ariño, Miguel A. (1997), La evolución del PIB en España. Documento de investigación. IESE Business School, Pamplona.

Arrighi, Giovanni (2008), Adan Smith en Pekín. Editorial Akal, Madrid.

Berzosa, Carlos (2009), “Crisis financiera, crisis global”, El País, 20 de febrero de 2009. Madrid. Disponible en: http:/www.elpais.com/articulo/ opinion/Crisis/financiera/crisis/global/elpepiopi/20090210elpepiopi_4/Tes/

Campuzano, José Luis (2007), “Una crisis de confianza”, Cinco Días, 18 de agosto de 2007, Madrid, España. Disponible en: http:// www.cincodias.com/articulo/opinion/crisis-confianza/ 20070818cdscdiopi_1/cdsopi/

De la Osa Bonilla, José Ramón (1992), “El Sistema Monetario Europeo (1985-1992): España y la devaluación de la peseta”. Escuela Universitaria de Estudios Empresariales. Universidad Complutense de Madrid, Madrid.

Harvey, David (1982), Limits to capital. Basil Blackwell, Oxford.

Ídem (2004), “El “nuevo” imperialismo: acumulación por desposesión”. Socialist Register 2004, pp. 99-129. CLACSO, Consejo Latinoamericano de Ciencias Sociales, Ciudad Autónoma de Buenos Aires.

Ídem (2007), “La solución espacial: Hegel, Von Thünen y Marx”, en Espacios del Capital, Akal, Cuestiones de Antagonismo n 44. Madrid.

Kondratiev, Nikolai (1925), The major Economic Cycles.

Lefebvre, Henri (1976), The survival of Capitalism: Reproduction of the Relations of Production: St Martin's Press, 1976, New York.

Lenin, Vladímir Ilich (1916), El imperialismo, fase superior del capitalismo. Edición electrónica Buenos Aires 2004. http:// www.laeditorialvirtual.com.ar/Pages/Lenin/Lenin_Imperialismo FaseCapitalismo_01.htm

Lipietz, Alain (1979), El capital y su espacio. Siglo XXI Editores, México. Del original francés : Le capital et son espace, (1977), F. Maspéro, París.

Ídem (1990), “Une approche regulationniste sur le futur de l'ecologie urbaine”. Konferenz Die Zukunft des Städtischen. Stadträum, Francfurt.

Ídem (1997), “El mundo del postfordismo”. Ensayos de economía n 12, vol. 7, julio 1997. Medellín. 
López, Isidro y Rodríguez, Emmanuel (2010), Fin de ciclo. Financiarización, territorio y sociedad de propietarios en la onda larga del capitalismo hispano (1959-2010). Editorial Traficantes de Sueños. 505 pp. Disponible en : http://www.traficantes.net/index.php/trafis/editorial/catalogo/utiles/fin_de_ciclo_financiarizacion_territorio_ y_sociedad_de_propietarios_en_la_onda_larga_del_capitalismo_hispano_1 959_2010

Meadows, Donella H.; Meadows, Dennis; Randers, Jorgen; Behrens, William (1975), Los Límites Del Crecimiento. Informe Al Club De Roma Sobre El Predicamento De La Humanidad. Fondo de Cultura Económica, México D. F.

Ministerio de Fomento (2009), Anuario estadístico de España.

Montaner, Josep María (2003), “Tendencias: La ciudad, ¿museo o parque temático?”, 19-11-2003, en la Vanguardia, Barcelona.

Muñoz, Francesc (2004), UrBANALització. La producció residencial de baixa densitat a la provincia de Barcelona, 1985-2001. Tesi Doctoral dirigida pel Drs. Ignasi de Solá-Morales i Rosa Ascón, Departament de Geografia, Universitat Autònoma de Barcelona. 3 vols.

Observatorio Metropolitano (2009), Manifiesto por Madrid. Crítica y crisis del modelo metropolitano. Editorial Traficantes de Sueños, Madrid.

Observatorio de la Sostenibilidad en España - OSE (2009), Sostenibilidad en España 2009. Editorial Mundiprensa, Madrid.

Rodríguez López, Julio (2008), “El año de los abundantes peligros económicos y de la crisis del mercado de la vivienda" en CIUDAD Y TERRITORIO Estudios Territoriales, XL, Tercera Época núm. 157, otoño 2008, Madrid. Pp. 551-561.

Rogoff, Kenneth (2009), “¿De la crisis financiera a la crisis de la deuda?”, El País, 13 de septiembre de 2009, Madrid. Disponible en: http:// www.elpais.com/articulo/primer/plano/crisis/financiera/crisis/deuda/ elpepueconeg/20090913elpneglse_10/Tes

Schumpeter, Joseph (1939), "Business Cycles: A theoretical, historical and statistical analysis of theCapitalist process”. McGraw-Hill Book Company, New York ,Toronto, London. Disponible en Les classiques des sciences sociales, http://classiques.uqac.ca/

Vázquez Romero, Juan Antonio (2009), “Los aportes de Henri Lefebvre a la Geografía urbana. Un corpus Teórico para entender las nuevas espacialidades”. $12^{\circ}$ Encuentro de Geógrafos de América Latina, 3-7 abril de 2009, Montevideo. 
Wallerstein, Immanuel (2007), Geopolítica y geocultura: ensayos sobre el moderno sistema mundial. Editorial Kairós, Madrid.

Recibido: 09.01.2010

Aceptado: 03.12.2010 